\section{Sub-registro de nascimentos no município de Centro Novo do Maranhão, 2002}

\section{Under-registration of births in the municipality of Centro Novo do Maranhão, 2002}

\author{
Adílson Lopes Cardoso \\ Departamento de Saúde Pública \\ Faculdade de Medicina \\ Universidade Federal do Maranhão (UFMA) \\ Rua Barão de Itapary, 155 \\ 65020-070 - São Luís - MA \\ cardosolc@uol.com.br \\ Maria Idalina Marques Fernandes \\ Departamento de Saúde Pública \\ Faculdade de Medicina \\ Universidade Federal do Maranhão (UFMA) \\ Antônio Augusto Moura da Silva \\ Departamento de Saúde Pública \\ Faculdade de Medicina \\ Universidade Federal do Maranhão (UFMA) \\ Vânia Maria de Farias Aragão \\ Departamento de Saúde Pública \\ Faculdade de Medicina \\ Universidade Federal do Maranhão (UFMA)

\section{Raimundo Antonio da Silva} \\ Departamento de Saúde Pública \\ Faculdade de Medicina \\ Universidade Federal do Maranhão (UFMA)
}

\section{Resumo}

Apesar da importância do registro de nascimento para o cálculo de vários indicadores de saúde, o sub-registro de nascimentos ainda é muito elevado no Brasil: 31,5\% em 1995. Após a gratuidade do registro de nascimento, poucos estudos foram feitos para estimar o sub-registro de nascimentos, especialmente em áreas rurais, onde este é ainda mais elevado. Estudou-se, no município de Centro Novo do Maranhão, por inquérito domiciliar, amostra por conglomerados de 1.157 pessoas, com aplicação de questionário. Foram avaliados o percentual de subregistro em todas as idades, o tempo decorrido entre o nascimento e o registro, a situação do registro segundo algumas variáveis e os motivos para o não registro. O percentual de sub-registro foi 8,6\% (Intervalo de Confiança de $95 \%$ - 7,0\% a 10,3\%), sendo que $95 \%$ dos registros foram efetuados fora do prazo. O intervalo médio de tempo entre o nascimento e o registro foi de 13,1 anos. As coortes nascidas antes da lei da gratuidade do registro de nascimento foram registradas mais cedo $(0,7$ ano) do que as nascidas depois da lei da gratuidade (15,2 anos). Não se observou associação entre registro e sexo ou renda familiar mensal. Baixa escolaridade foi associada marginalmente a maiores percentuais de sub-registro e a maiores percentuais de registro fora do prazo. O subregistro foi mais elevado entre os menores de um ano (25\%), e o registro fora do prazo predominou entre adultos. Os principais motivos do não registro foram "falta de interesse dos pais" e "o pai não reconheceu a paternidade". O sub-registro foi menor do que o esperado devido à realização de "mutirões" de registro, que facilitaram o acesso ao mesmo, de forma gratuita, mesmo fora do prazo legal.

Palavras-chave: Nascidos vivos. Registro de nascimento. Sub-registro. 
Abstract

Despite the importance of birth registration for calculating many health indicators, under-registration of births is still very high in Brazil, 31.5\% in 1999. After birth registration became free, few studies were conducted to estimate the rate of unregistered births, especially in rural areas, where under-registration is much higher. This study was conducted during PITS (Program of Interiorization of Work in Health) activities in the municipality of Centro Novo do Maranhão, in a state with the highest rate of unregistered births. A cluster sample of 1,157 people was studied by means of a household survey using a questionnaire to estimate the rate of unregistered births, the time elapsed between the birth and its registration, to evaluate registration according to sex, age, schooling of the head of the family and family income, and reasons for not registering. The rate of unregistered births was 8.6\% (95\% Confidence Interval 7.0\% to $10.3 \%) .95 \%$ of the registrations were performed after the period within which it is legally required in rural areas (within 105 days of birth). The mean time between the birth and its registration was 13.1 years. Cohorts born after the registration became free were registered earlier $(0.7$ year) than those born before (15.2 years). There were no associations between registration and sex or family income. Low schooling was marginally associated with higher rates of under-registration and higher rates of registration after the period within which it is required. Under-registration was higher among under-one-yearolds (25\%) whereas registration after the period within which it is required predominated among adults. The main reasons for not registering were "lack of interest of the parents" and "the father did not recognize fatherhood". The rate of unregistered births was lower than expected due to mass campaigns that increased access to free registration, even after the period within which it is legally required.

Keywords: Live borns. Birth registration. Under-registration of births.

\section{Introdução}

O registro de nascimento, além de ser um documento pelo qual se dá o ingresso do indivíduo na sociedade civil, é importante para o cálculo de vários indicadores de saúde, dentre os quais se destaca o coeficiente de mortalidade infantil ${ }^{1}$. É fundamental que o registro seja feito logo após o nascimento para que não ocorram distorções no cálculo dos indicadores de saúde, para que estes indicadores possam ser úteis para o planejamento e a avaliação das ações de saúde ${ }^{2}$.

A Lei de Registros Públicos n 6.015 de 31 de dezembro de 1973, em seu artigo 50, determina que todo nascimento que ocorrer no território nacional deverá ser dado a registro, dentro do prazo de 15 (quinze) dias, que será ampliado em até 3 (três) meses para os lugares distantes mais de 30 (trinta) quilômetros da sede do cartório. Este prazo foi estabelecido no caso do pai ser o declarante. Caso o declarante seja a mãe ou outro responsável, o prazo é prorrogado por 45 (quarenta e cinco) dias, sendo, portanto, de 15 (quinze) mais 45 (quarenta e cinco) dias ou, havendo distância maior de 30 (trinta) quilômetros da sede do cartório, de 3 (três) meses mais quinze dias ${ }^{3}$.

Apesar da sua importância, no Brasil o sub-registro de nascimentos é elevado. No período 80-86, o sub-registro foi estimado, a partir dos dados da Pesquisa Nacional por Amostragem de Domicílios de 1994, em $26,0 \%$ para o Brasil, sendo de $43,5 \%$ para a região Nordeste e $12,7 \%$ para a região Sul ${ }^{4}$. Estimativas mais recentes, a partir de dados do Registro Civil de 1995, calcularam o subregistro de nascimentos em $31,8 \%$ para o Brasil, tendo o Estado do Maranhão apresentado o maior percentual, de $84,3 \%$, e o Distrito Federal o menor ${ }^{5}$.

Outros trabalhos realizados em diferentes localidades brasileiras também estimaram o sub-registro de nascimentos de menores de 1 ano, observando-se percentuais maiores na região Nordeste e menores na região Sul. O sub-registro foi de $32 \%$ em Salvador (BA), em 1977 , 7,8\% em Pelotas (RS), em 1982 7 , 68,4\% em Piripiri (PI), em 1983/ 
$84^{8}, 17,98 \%$ em Natal (RN), em $1987^{9}, 9,1 \%$ em Maringá, em $1989^{10}$ e 5,3\% em Londrina (PR), em 1994².

No Maranhão, o sub-registro de nascimentos e de óbitos é elevado ${ }^{5,11}$. Em trabalho feito em um bairro de São Luís, Madre de Deus, em 1993, o sub-registro de nascimentos em menores de 5 anos foi estimado em $18,9 \%^{12}$. Dentre as razões para isto estão $\mathrm{o}$ alto percentual de partos domiciliares observados no Estado, estimado em 22,6\% em $1996^{13}$, a existência de cemitérios clandestinos, o custo do registro elevado para uma população de baixo poder aquisitivo e a grande distância entre muitas áreas rurais e os cartórios, em um Estado que até antes do censo de 2000 tinha a maioria de sua população morando em áreas rurais.

Um dos obstáculos para o registro, o custo, foi abolido com a lei da gratuidade do registro de nascimento e óbito, no. 9534, de 10 de dezembro de $1997^{14}$. Há poucos trabalhos recentes que possam verificar o efeito desta mudança no registro de nascimentos. Raros também são os trabalhos realizados em áreas rurais remotas e em municípios novos, recém-emancipados. Poucos também são os trabalhos que estudam o subregistro em toda a população e não apenas nos menores de um ano.

$O$ presente trabalho foi realizado em um município novo, recém-emancipado, Centro Novo do Maranhão, com população predominantemente rural, como parte das atividades do Programa de Interiorização do Trabalho em Saúde (PITS). Teve como objetivos: estimar o sub-registro de nascimentos em uma amostra aleatória de residentes de todas as idades, quantificar o percentual de registros que ocorreram fora do prazo legal, estimar o intervalo de tempo entre o nascimento e o registro de nascimento, avaliar a situação do registro segundo sexo, idade, escolaridade do chefe de família e renda familiar, e identificar os motivos do não registro.

\section{Metodologia}

O município de Centro Novo do Maranhão tinha uma população de 15.096 habi- tantes em 2002 , sendo que $78,5 \%$ residiam na zona rural e $21,5 \%$ na zona urbana. Possui sete povoados e dois distritos rurais, conhecidos como Chega Tudo e Limão, distantes da sede aproximadamente 38 quilômetros. A ligação dos distritos e povoados com a sede do município se dá por estrada de difícil acesso, principalmente na época das chuvas.

$\mathrm{O}$ inquérito domiciliar por amostragem foi o método utilizado para realização desta pesquisa, onde foi estudada amostra aleatória por conglomerados de 1.157 pessoas, sorteadas de aproximadamente $70 \%$ da população do município de Centro Novo do Maranhão (sede, distritos do Chega Tudo e Limão). Cerca de $30 \%$ dos domicílios foram excluídos do sorteio, pois os mapas das áreas rurais dispersas não puderam ser obtidos. $\mathrm{O}$ tamanho amostral foi estimado em 997 nascimentos para a estimativa de uma proporção, considerando-se $3 \%$ de precisão relativa, erro tipo I de $5 \%$, para uma prevalência máxima de 50\%. Para a comparação de proporções, considerando-se erro tipo I de 5\%, poder do estudo de $80 \%$, prevalência de $50 \%$ e diferença mínima a ser detectada entre proporções de 6\%, o tamanho amostral estimado foi de 1.119 nascimentos ${ }^{15}$. Foram realizadas entrevistas em 600 domicílios. No primeiro estágio foram sorteadas 30 quadras, sendo duas quadras na sede do Município, sete quadras no distrito do Limão e 21 quadras no distrito do Chega Tudo, o mais populoso. No segundo passo foram sorteados 20 domicílios para visitação em cada quadra. O ponto de partida de cada quadra foi escolhido colocando-se letras nas esquinas e sorteando-se uma esquina, prosseguindo-se a partir daí sempre em sentido horário. No terceiro estágio foram sorteadas duas pessoas em cada domicílio, perfazendo uma amostra de 1.157 pessoas. A amostra deveria incluir 1.200 pessoas, mas em algumas casas existia somente um morador e alguns questionários com preenchimento incompleto foram descartados.

Na coleta de dados utilizou-se entrevista semi-estruturada, tendo como instrumento um questionário. A coleta foi realizada por 14 agentes comunitários de saúde previamente treinados pelos pesquisadores, integrantes das 
equipes do PITS. O questionário incluiu as seguintes variáveis: data de nascimento, sexo, se foi registrado em cartório, local e data do registro, escolaridade do chefe de família, renda familiar mensal e motivo do não registro. O instrumento comprobatório do registro foi a certidão de nascimento. Na ausência desta, não foram preenchidos os itens referentes ao local e data do registro. As pessoas que disseram ser registradas, mas não mostraram a certidão de nascimento, foram consideradas como não registradas. Para a renda familiar foram considerados todos os rendimentos de todas as pessoas residentes no domicílio que de algum modo contribuíam para o sustento da família.

O processo de apuração e tabulação dos dados foi feito por computador, utilizandose o programa Epi-Info ${ }^{16}$. Na análise estatística, utilizou-se o qui-quadrado para comparar proporções e análise de variância para comparar médias. O nível de significância adotado foi de 0,05 . Intervalo de confiança de $95 \%$ foi calculado assumindo-se distribuição binomial.

\section{Resultados}

Das 1.157 pessoas entrevistadas, 1.058 $(91,4 \%)$ foram registradas e mostraram o registro de nascimento para o entrevistador. O sub-registro de nascimento foi baixo, de 8,6\% - Intervalo de Confiança de 95\% - 7,0\% a $10,3 \%)$, pois 60 pessoas $(5,2 \%)$ não possuíam a certidão de nascimento e outras 39 $(3,6 \%)$ disseram possuir, mas não apresentaram certidão de nascimento no momento da entrevista. Entretanto, dentre as registradas apenas 53 (5,0\%) o foram no prazo legal, ou seja, até 105 dias após o nascimento. Entre os menores de um ano que tinham mais de 105 dias de vida, o percentual de subregistro foi de $25 \%$. O intervalo médio de tempo entre o nascimento e o registro de nascimento foi de 13,1 anos.

A metade das pessoas entrevistadas possuíam renda familiar mensal de até meio salário mínimo, e apenas 2,6\%, ganhavam acima de dois salários mínimos. A renda familiar e sexo não estiveram associados com registro de nascimento (Tabelas 1 e 2).

Tabela 1 - Registro de nascimento segundo renda familiar no município de Centro Novo do Maranhão, 2002.

Table 1 - Birth registration according to family income in the municipality of Centro Novo do Maranhão, 2002.

\begin{tabular}{lrrrrrrrr}
\hline $\begin{array}{l}\text { Renda familiar } \\
\text { (salários mínimos) }\end{array}$ & \multicolumn{2}{c}{ Sem registro } & \multicolumn{2}{c}{ Até 105 dias } & \multicolumn{2}{c}{ Acima de 105 dias } & \multicolumn{2}{c}{ Total } \\
\hline Até $1 / 2$ & & $\%$ & $\mathrm{n}$ & $\%$ & $\mathrm{n}$ & $\%$ & $\mathrm{n}$ & $\%$ \\
\hline $1 / 2$ a 1 & 56 & 9,7 & 27 & 4,7 & 495 & 85,6 & 578 & 50,0 \\
1 a 2 & 33 & 8,0 & 20 & 4,8 & 362 & 87,2 & 415 & 35,9 \\
Mais de 2 & 6 & 4,5 & 6 & 4,5 & 122 & 91,0 & 134 & 11,5 \\
\hline Total & 4 & 13,3 & - & - & 26 & 89,7 & 30 & 2,6 \\
\hline$\chi^{2}=6.26$ P=0.394 & 99 & 8,6 & 53 & 4,6 & 1055 & 86,9 & 1157 & 100,0 \\
\hline
\end{tabular}

Tabela 2 - Registro de nascimento segundo sexo no município de Centro Novo do Maranhão, 2002.

Table 2 - Birth registration according to sex in the municipality of Centro Novo do Maranhão, 2002.

\begin{tabular}{lcrrrrrrr}
\hline Sexo & \multicolumn{2}{c}{ Sem registro } & \multicolumn{2}{c}{ Até 105 dias } & \multicolumn{2}{c}{ Acima de 105 dias } & \multicolumn{2}{c}{ Total } \\
& $\mathrm{n}$ & $\%$ & $\mathrm{n}$ & $\%$ & $\mathrm{n}$ & $\%$ & $\mathrm{n}$ & $\%$ \\
\hline Feminino & 48 & 8,1 & 25 & 4,2 & 520 & 87,7 & 593 & 51,3 \\
Masculino & 51 & 9,0 & 20 & 5,0 & 485 & 86,0 & 564 & 48,7 \\
\hline Total & 99 & 8,6 & 53 & 4,6 & 1005 & 86,9 & 1157 & 100,0 \\
\hline$\chi^{2}=0.75 \mathrm{P}=0.686$ & & & & & & & &
\end{tabular}


O chefe de família era analfabeto ou tinha instrução elementar em $56,2 \%$ dos casos. Observou-se associação marginalmente significante (valor de P entre 0,10 e 0,05) entre baixa escolaridade e maiores percentuais de sub-registro e menores percentuais de registro dentro do prazo legal (Tabela 3).

Observou-se associação entre idade e registro. Os menores de um ano tiveram o maior percentual de sub-registro e os menores percentuais de registro fora do prazo, enquanto os maiores de 20 anos tiveram os menores percentuais de sub-registro e os maiores percentuais de registro fora do prazo (Tabela 4).

Não houve diferença no intervalo médio de tempo entre o nascimento e o registro, de acordo com a renda familiar $(\mathrm{P}=0,430)$ ou o sexo $(\mathrm{P}=0,428)$. O intervalo médio de tempo entre o nascimento e o registro foi de 15,0 anos para famílias de chefes analfabetos ou com instrução elementar, e de 11,6 anos para aquelas cujo chefe cursou o ensino médio $(\mathrm{P}=0,014)$. Quanto maior era idade, maior foi o intervalo médio de tempo entre o nascimento e o registro $(\mathrm{P}<0.001)$. $\mathrm{O}$ intervalo médio entre o nascimento e o registro foi de 0,7 anos para as coortes nascidas antes da lei da gratuidade (até 1997) e de 15,2 anos para as coortes nascidas depois da lei da gratuidade (de $1998 \mathrm{em}$ diante), com diferença altamente significante entre as médias $(\mathrm{P}<0,001)$.

Predominaram entre os motivos citados para o não registro "falta de interesse dos pais" e "o pai não reconheceu a paternida-

Tabela 3 - Registro de nascimento segundo escolaridade do chefe de família no município de Centro Novo do Maranhão, 2002.

Table 3 - Birth registration according to schooling of the head of the family in the municipality of Centro Novo do Maranhão, 2002.

\begin{tabular}{lrrrrrrrr}
\hline Escolaridade* $^{*}$ & \multicolumn{2}{c}{ Sem registro } & \multicolumn{2}{c}{ Até 105 dias } & \multicolumn{2}{c}{ Acima de 105 dias } & \multicolumn{2}{c}{ Total } \\
& $\mathrm{n}$ & $\%$ & $\mathrm{n}$ & $\%$ & $\mathrm{n}$ & $\%$ & $\mathrm{n}$ & $\%$ \\
\hline Analfabeto/Elementar & 65 & 10,1 & 27 & 4,2 & 553 & 85,7 & 645 & 56,2 \\
Ensino fundamental & 30 & 6,5 & 21 & 4,6 & 408 & 88,9 & 459 & 40,0 \\
Ensino médio & 3 & 6,8 & 5 & 11,9 & 36 & 81,9 & 44 & 3,8 \\
\hline Total & 98 & 8,6 & 53 & 4,6 & 997 & 86,9 & 1148 & 100,0 \\
\hline
\end{tabular}

$\chi^{2}=9.16 \mathrm{P}=0.057$

*Excluídos nove casos com informação ignorada. Nine cases with missing information were excluded.

Tabela 4 - Registro de nascimento segundo idade no município de Centro Novo do Maranhão, 2002.

Table 4 - Birth registration according to age in the municipality of Centro Novo do Maranhão, 2002.

\begin{tabular}{lrrrrrrrr}
\hline Idade (anos) & \multicolumn{2}{c}{ Sem registro } & \multicolumn{2}{c}{ Até 105 dias } & \multicolumn{2}{c}{ Acima de 105 dias } & \multicolumn{2}{c}{ Total } \\
& $\mathrm{n}$ & $\%$ & $\mathrm{n}$ & $\%$ & $\mathrm{n}$ & $\%$ & $\mathrm{n}$ & $\%$ \\
\hline 0 a 1 & $18 *$ & 35,3 & 22 & 43,1 & 11 & 21,6 & 51 & 4,4 \\
1 a 10 & 40 & 11,7 & 14 & 4,1 & 289 & 84,6 & 307 & 29,6 \\
11 a 20 & 17 & 5,5 & 14 & 4,6 & 276 & 89,9 & 343 & 26,3 \\
Mais de 20 & 24 & 5,2 & 3 & 0,7 & 429 & 94,1 & 456 & 39,4 \\
\hline Total & 99 & 8,6 & 53 & 4,6 & 1005 & 86,9 & 1157 & 100,0 \\
\hline
\end{tabular}

$\chi^{2}=264.90 \mathrm{P}=0.001$

* Sete (13.7\%) crianças não tinham registro, mas tinham até 105 dias de vida, estando dentro do prazo legal para registro de nascimento. Seven children did not have birth register but were up to 105 day old, below the legal limit for birth register. 
Tabela 5 - Motivos para a falta de registro de nascimento no município de Centro Novo do Maranhão, 2002.

Table 5 - Reasons for not registering birth in the municipality of Centro Novo do Maranhão, 2002.

\begin{tabular}{lrc} 
Motivos & $\mathrm{n}$ & $\%$ \\
\hline Falta de interesse & 18 & 18,2 \\
Não está com o registro em casa & 18 & 18,2 \\
Perda do registro & 13 & 13,1 \\
Pais não tinham documento & 12 & 12,1 \\
Pai não reconheceu a paternidade & 14 & 10,1 \\
Fez, mas o cartório não entregou & 8 & 8,1 \\
Falta de oportunidade & 6 & 6,1 \\
Outros & 14 & 14,1 \\
\hline Total & 99 & 100,0 \\
\hline
\end{tabular}

de". A "falta de documento dos pais" também foi outra causa importante da omissão do registro. Dentre os que afirmaram ter se registrado, mas não mostraram o documento no momento da entrevista as razões apontadas foram "perda do registro", "não estava com o registro em casa" e "fez, mas o cartório não entregou".

\section{Discussão}

O percentual de sub-registro foi de $8,6 \%$, sendo que $95 \%$ dos registros foram efetuados fora do prazo. $\mathrm{O}$ intervalo médio de tempo entre o nascimento e o registro de nascimento foi de 13,1 anos. Não se observou associação entre registro e sexo ou renda familiar mensal em salários mínimos. Baixa escolaridade associou-se marginalmente a maiores percentuais de sub-registro e a maiores percentuais de registro fora do prazo. $\mathrm{O}$ sub-registro foi mais prevalente entre os menores de um ano, enquanto o registro fora do prazo predominou entre adultos. Coortes nascidas antes da lei da gratuidade tiveram menor tempo médio entre o nascimento e o registro. Os principais motivos do não registro foram "falta de interesse dos pais", "o pai não reconheceu a paternidade".

$O$ percentual de sub-registro foi bem inferior ao esperado, apesar de o município se encontrar a mais de $100 \mathrm{~km}$ do cartório mais próximo. Esse fato se explica pois, antes da realização dessa pesquisa, em 2001 e 2002, foram feitos três mutirões para registro de nascimento no Município, onde foram registradas 1.119 pessoas. Com os mutirões, as pessoas puderam ter o registro de nascimento efetuado no próprio município, não tendo que arcar com os custos de transporte para o cartório localizado em outro município. A falta do registro estava também dificultando o cadastro dos usuários para o cartão SUS.

Não houve relação entre renda familiar e registro de nascimento, achado esse diferente do encontrado por Campelo ${ }^{12}$, que observou relação entre essas variáveis, ao estudar o sub-registro em um bairro de São Luís em 1993. Isto sugere que, após a gratuidade do registro de nascimento houve uma redução muito grande do sub-registro de nascimentos neste município rural do Maranhão, pois muitos cidadãos aproveitaram para fazer o seu registro por ocasião dos mutirões. Tal achado corrobora a hipótese de que o custo do registro era, provavelmente, o principal fator para a omissão do registro. Com a gratuidade, registros fora do prazo também foram estimulados. Isto explica o elevado tempo médio observado entre o nascimento e o registro e o percentual extremamente elevado de registro fora do prazo nesta população. Entretanto, o subregistro em menores de um ano ainda permanece elevado. Um dos possíveis fatores intervenientes, apesar de não mencionado expressamente pelos entrevistados, é a dis- 
tância entre a sede do município e o cartório mais próximo.

Não houve associação significativa entre sexo e registro de nascimento. Outros trabalhos realizados também não observaram esta associação $0^{2,6,8,12}$. Observou-se associação marginalmente significante entre escolaridade do chefe de família e registro de nascimento. Em trabalhos realizados por Mello Jorge et al. ${ }^{2}$ (1997) e Cunha ${ }^{9}$ (1991), esta associação também foi observada, o que sugere que a informação e fatores culturais são hoje os fatores mais relacionados com a omissão do registro e com o registro fora do prazo, após a lei da gratuidade dos registros.

Houve associação significante entre registro de nascimento e idade. A maioria dos entrevistados foram registrados tardiamente após os 10 anos de idade. Embora atualmente a maioria das crianças menores de um ano estejam sendo registradas mais cedo, o percentual de sub-registro em menores de um ano ainda é alto e muitas destas crianças ainda são registradas fora do prazo.

Os mutirões para registro de nascimento têm alcançado bons resultados, garantindo o acesso a esse registro gratuitamente, mesmo fora do prazo legal e colaborando para que os adultos regularizem sua situa- ção de registro civil. É importante a realização de campanhas de esclarecimento junto à comunidade, mostrando a importância desse registro e de se aproveitar a oportunidade dos mutirões para efetuar o registro de nascimento, visto que o registro de nascimento é o primeiro passo para a cidadania.

Em conclusão, o percentual de sub-registro foi bem inferior ao esperado, pois somente $8,6 \%$ não possuíam o registro no momento da entrevista. Entretanto, o percentual de registros fora do prazo foi muito elevado, $95 \%$. O registro de nascimento não esteve associado com o sexo ou com a renda familiar, porém maiores percentuais de sub-registro foram encontradas entre os analfabetos e com instrução elementar e nos menores de um ano. Neste município novo, recém-emancipado, localizado no meio rural maranhense, a gratuidade do registro associada aos mutirões permitiu que os cidadãos, mesmo com muito atraso, tivessem o seu registro de nascimento efetuado. Apenas a gratuidade parece não ter tido a repercussão desejada neste município rural, cujo cartório mais próximo está a mais de $100 \mathrm{~km}$ de distância, sendo a associação da gratuidade com os mutirões efetiva para a diminuição do sub-registro de nascimentos.

\section{Referências}

1. Laurenti R, Mello Jorge MHP, Lebrão ML, Gotlieb SLD. Registro dos eventos vitais. In: Laurenti R, Mello Jorge MHP, Lebrão ML, Gotlieb SLD. Estatísticas de Saúde. São Paulo: Epu/Edusp; 1985. p. 39-76.

2. Mello Jorge MHP, Gotlieb SLD, Andrade SM. Análise dos registros de nascimentos vivos em localidade urbana no Sul do Brasil. Rev Saúde Pública 1997; 31: 78-89.

3. Brasil. Lei de Registros Públicos. Lei no 6015, de 31 de dezembro de 1973. Disponível em http:// www.soleis.adv.br/registrospublicos.htm

4. Simões CC. Novas estimativas da mortalidade infantil - 1980/87. In: Simões CC, org. Perfil estatístico de crianças e mães no Brasil: mortalidade infantil e saúde na década de 80. Rio de Janeiro: IBGE; 1989. p. 14-48.

5. Simões CC. Estimativas da mortalidade infantil por microrregiões e municípios. Brasília: Ministério da Saúde; 1999.
6. Almeida MMG. Subregistro de nascimento em Salvador - Bahia/Brasil. Rev Saúde Pública 1979; 13: 208-19.

7. Barros FC, Victora CG, Teixeira AMB, Puerto Filho M. Mortalidade perinatal e infantil em Pelotas, Rio Grande do Sul: nossas estatísticas são confiáveis? Cad Saúde Pública 1985; 1: 348-58.

8. Portela MHRB. Sub-registro de nascimentos vivos em localidade do Estado de Piauí, Brasil. Rev Saúde Pública 1989; 23: 493-501.

9. Cunha VF. O sub-registro de nascimento e os fatores que contribuem para a omissão do registro [tese de doutorado]. São Paulo: Faculdade de Saúde Pública da USP; 1991.

10. Souza RKT, Gotlieb SLD. Sub-registro de nascimentos vivos hospitalares em área urbana da região sul do Brasil, em 1989. Rev Saúde Pública 1993; 27: 177-84. 
11. Paes NA, Albuquerque MEE. Avaliação da qualidade dos dados populacionais e cobertura dos registros de óbitos para as regiões brasileiras. Rev Saúde Pública 1999; 33: 43.

12. Campelo IC, Costa LMM, Pinheiro LM, Silva, MJM. Registro de nascidos vivos no bairro Madre de Deus São Luis - MA [Monografia de Especialização em Saúde Pública]. São Luís: Universidade Federal do Maranhão; 1993.

13. Coimbra LC, Mochel EG. Assistência pré e perinatal. In: Tonial SR, Silva AAM, org. Saúde, nutrição e mortalidade infantil no Maranhão. São Luís: UFMA/ Secretaria de Estado da Saúde/Unicef; 1997. p. 30-5.
14. Brasil. Lei da Gratuidade do Registro de Nascimento e Óbito. Lei no. 9534, de 10 de dezembro de 1997.

Disponível em http://www.soleis.adv.br/

registronascimentoobitogratuidade.htm

15. Lwanga SK, Lemeshow S. Sample size determinations in health studies. Geneva: World Health Organization; 1991.

16. Dean AG, Arner TG, Sangam S, Sunki GG, Friedman R, Lantinga $\mathrm{M}$ et al. Epi Info 2000: a database and statistics program for public health professionals for use on Windows 95, 98, NT, and 2000 computers. Atlanta, Georgia, USA: Centers of Disease Control and Prevention; 2000.

Recebido em: 02/08/2002

Versão final reapresentada em: 11/01/2003

Aprovação em: 09/06/2003 